\title{
Supporting Information for Dynamic Effects and Hydrogen Bonding in Mixed-Halide Perovskite Solar Cell Absorbers
}

Regan G. Wilks, ${ }^{1 *}$ Axel Erbing, ${ }^{2}$ Golnaz Sadoughi, ${ }^{3}$ David E. Starr, ${ }^{1}$ Evelyn Handick, ${ }^{1}$ Frank Meyer, ${ }^{4}$ Andreas Benkert, ${ }^{4,5}$ Marcella Iannuzzi, ${ }^{6}$ Dirk Hauschild, ${ }^{5,7,8}$ Wanli Yang, ${ }^{9}$ Monika Blum, ${ }^{8,9,10}$ Lothar Weinhardt, ${ }^{5,7,8}$ Clemens Heske, ${ }^{5,7,8}$ Henry J. Snaith, ${ }^{3}$ Michael Odelius, ${ }^{2 *}$ and Marcus Bär ${ }^{1,11,12}$

${ }^{1}$ Renewable Energy, Helmholtz-Zentrum Berlin für Materialien und Energie GmbH (HZB), Germany

${ }^{2}$ Department of Physics, Stockholm University, AlbaNova University Center, Stockholm, Sweden

${ }^{3}$ Department of Physics, Clarendon Laboratory, University of Oxford, UK

${ }^{4}$ Experimental Physics 7, University of Würzburg, Germany

${ }^{5}$ Institute for Photon Science and Synchrotron Radiation (IPS), Karlsruhe Institute of Technology (KIT), Germany

${ }^{6}$ Physical Chemistry Institute, University of Zürich, Zürich, Switzerland

${ }^{7}$ Institute for Chemical Technology and Polymer Chemistry (ITCP), Karlsruhe Institute of Technology (KIT), Germany

${ }^{8}$ Department of Chemistry and Biochemistry, University of Nevada, Las Vegas (UNLV), USA

${ }^{9}$ Advanced Light Source, Lawrence Berkeley National Laboratory, USA

${ }^{10}$ Chemical Sciences Division, Lawrence Berkeley National Laboratory, USA ${ }^{11}$ HelmholtzInstitute Erlangen-Nürnberg for Renewable Energy (HI ERN), Berlin, Germany

${ }^{12}$ Department of Chemistry and Pharmacy, Friedrich-Alexander-Universität Erlangen-Nürnberg (FAU), Erlangen, Germany

\section{Corresponding Authors}

Regan G. Wilks regan.wilks@helmholtz-berlin.de

Michael Odelius odelius@fysik.su.se 


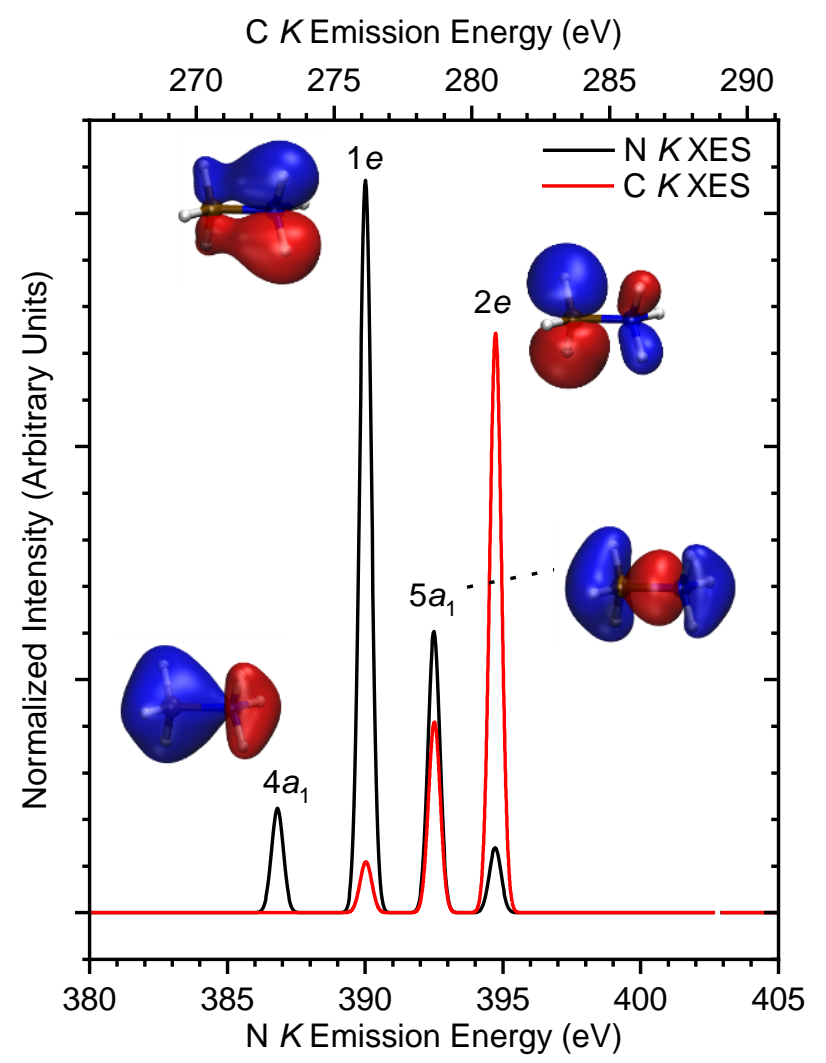

Figure S1. Calculated N (blue) and C (red) $K$ XES spectra of isolated $\mathrm{CH}_{3} \mathrm{NH}_{3}{ }^{+}$. Isosurfaces of the orbitals corresponding to the respective transitions are also shown.

The simulated $\mathrm{C}$ and $\mathrm{N} K$ XES spectra of gas-phase $\mathrm{CH}_{3} \mathrm{NH}_{3}{ }^{+}$are shown in Figure $\mathrm{S} 1$, along with isosurfaces of the orbitals corresponding to each transition. The simulated spectra are very similar to the respective $\mathrm{CH}_{3} \mathrm{NH}_{3} \mathrm{PbI}_{3}$ results and are used in the main text (and below) to describe and discuss those results. 

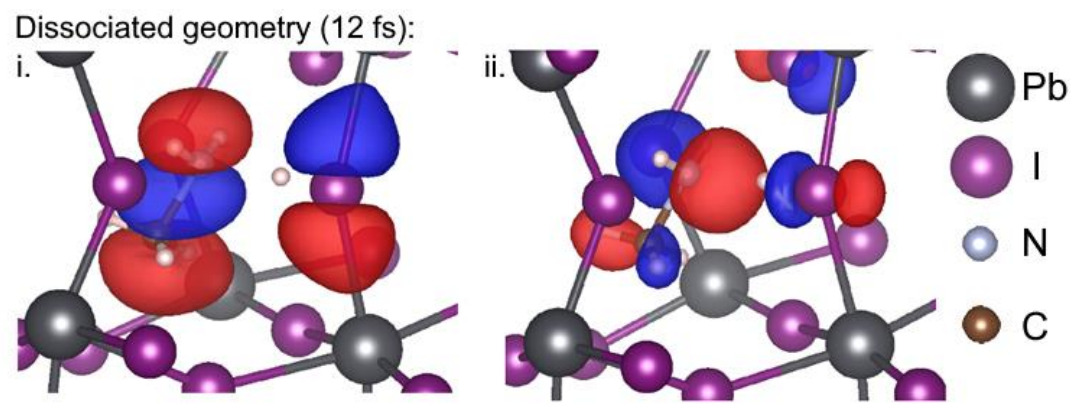

Ground-state geometry (0 fs):
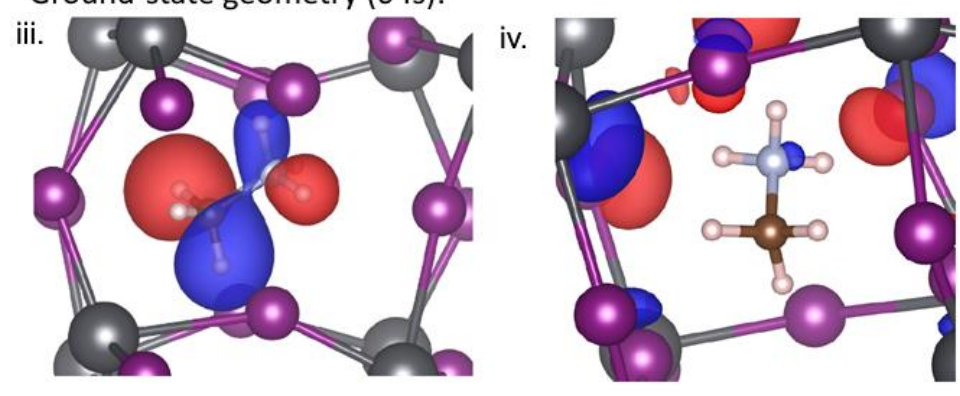

Figure S2. Selected orbitals in excited/dissociated geometry (i and ii) and ground-state geometry (iii and iv). The $5 a_{1}$ orbital shape (i) in dissociated geometry shows significant electron density on both the $\mathrm{CH}_{3} \mathrm{NH}_{2}----\mathrm{H}^{+}$moiety and the I sites. The orbital shown in (ii) is a three-center N-H-I anti-bonding orbital formed by dynamically-induced hybridization of the ground state $2 \mathrm{e}$ (iii) and H-bonding (iv) orbitals. 


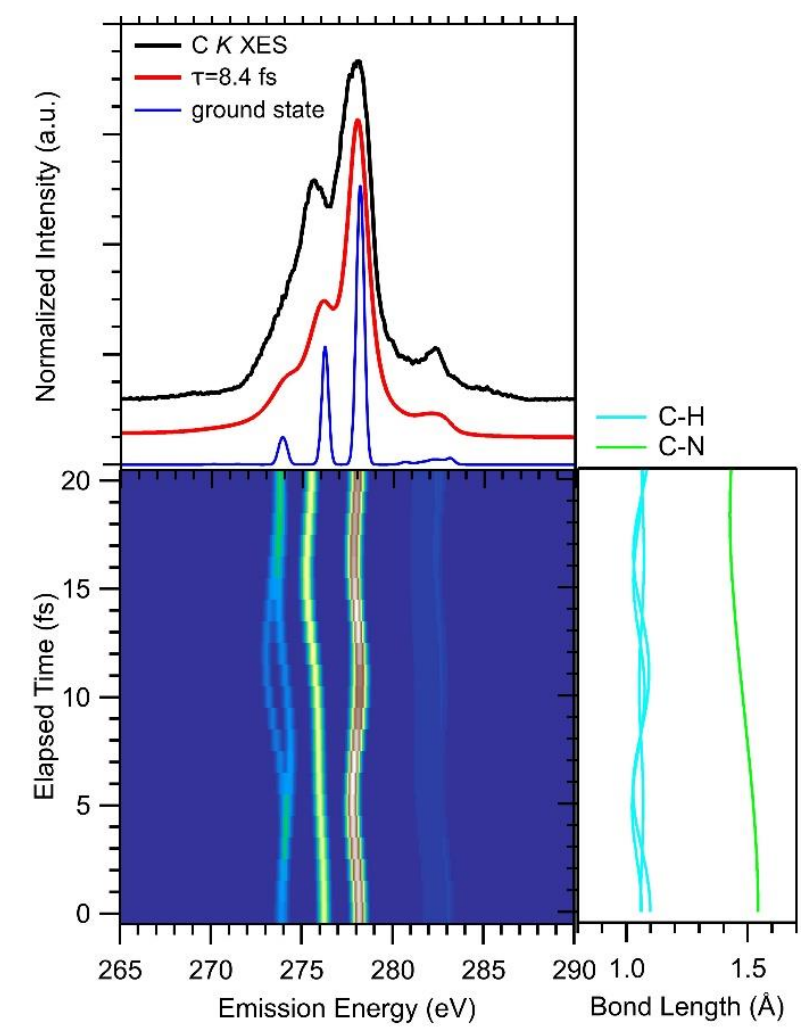

Figure S3. Calculations of $\mathrm{CH}_{3} \mathrm{NH}_{3} \mathrm{PbI}_{3} \mathrm{C} \mathrm{K}$ XES spectra as a function of elapsed time since core excitation. Top panel shows the ground-state ( $0 \mathrm{fs}$ ) calculation (blue), the Gaussian-Lorentzianbroadened $\tau=8.4$ fs spectrum (red), and the measured (nonresonant) C K XES spectrum (black). Spectra in the top panel were normalized and stacked for clarity. The panel at the right shows the $\mathrm{C}-\mathrm{N}$ and $\mathrm{C}-\mathrm{H}$ bond lengths as a function of time after excitation.

The calculated dynamic $\mathrm{C} K$-edge XES spectra are shown in Figure S3. The effects of ultrafast dynamics on the spectra are much smaller than in the corresponding $\mathrm{N} K$ XES spectra. There is good agreement between the result of the modelling (individual Gaussian-broadened contributions were added exponentially with a time constant of $8.4 \mathrm{fs}$, then Lorentzian broadening was added) and the spectrum measured with nonresonant $\left(\mathrm{h} v_{\mathrm{exc}}=320 \mathrm{eV}\right)$ excitation. The main features in the calculated spectrum can be inferred from the main text discussion of the N $K$ XES: The main peak at $278.2 \mathrm{eV}$ is the weakly antibonding orbital dominated by the $-\mathrm{CH}_{3} 1 e$ contribution, and the 
weaker peak at $274 \mathrm{eV}$ corresponds to the weakly bonding orbital with mainly $-\mathrm{NH}_{3}{ }^{+}$character. The feature at $276.3 \mathrm{eV}$ is the $-\mathrm{CH}_{3} 5 a_{1}$. The right panel of Figure $\mathrm{S} 3$ shows that the bond lengths are only slightly perturbed by the $\mathrm{C} 1 \mathrm{~s}$ core ionization. The dynamic effects in the C XES are mostly related to a slight contraction of the $\mathrm{C}-\mathrm{N}$ bond, and the most distinctive effect is the splitting of the $5 a_{1}$ orbital in the 6-14 fs range.

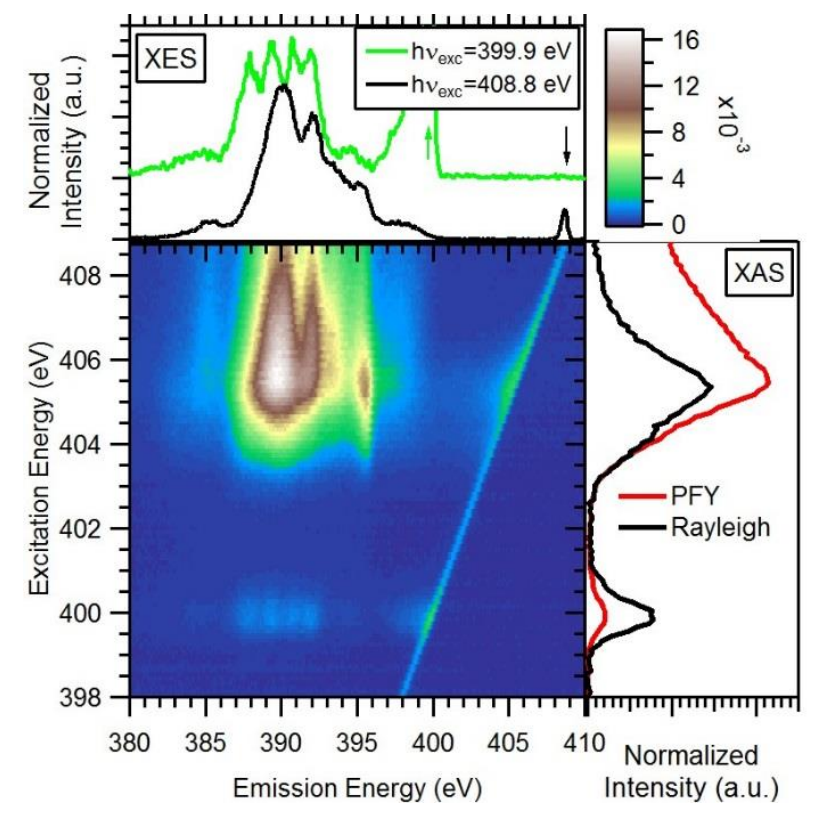

Figure S4. Main panel: $\mathrm{N}$ K-edge RIXS map of $\mathrm{CH}_{3} \mathrm{NH}_{3} \mathrm{PbI}_{3-\mathrm{x}} \mathrm{Cl}_{\mathrm{x}}$. Spectral intensity is displayed according to the color scale on the top-right. The top panel shows individual emission spectra excited well above the absorption edge $(408.8 \mathrm{eV})$ and at the center of the pre-edge resonance (399.9 eV), normalized to their maximum peak height (and offset for clarity). The absorption spectra in the right panel indicate the integral intensity from the main panel (red) and the intensity of the Rayleigh line (black). A uniform background signal was subtracted from both, and they were normalized to overlap at the absorption onset of the main edge.

The same RIXS map shown in the main text Figure 3c is reproduced in Figure S4, showing the entire measured excitation energy axis, including the pre-edge feature centered at $399.9 \mathrm{eV}$. The 
top panel compares the spectrum excited above resonance with the spectrum resonantly excited on the pre-edge. The shape and position of the features in the resonantly-excited spectra do not clearly match any part of the $\mathrm{CH}_{3} \mathrm{NH}_{3} \mathrm{PbI}_{3-\mathrm{x}} \mathrm{Cl}_{\mathrm{x}}$ spectrum or previously published results. The position of the resonance is similar to that previously ${ }^{1}$ attributed to an imine-containing $(\mathrm{R}-\mathrm{C}=\mathrm{NH})$ group. For the chosen scanning speed of $400 \mu \mathrm{m} / \mathrm{s}$, the maximum height of the pre-edge peak is approximately $10 \%$ of the intensity at the absorption resonance.

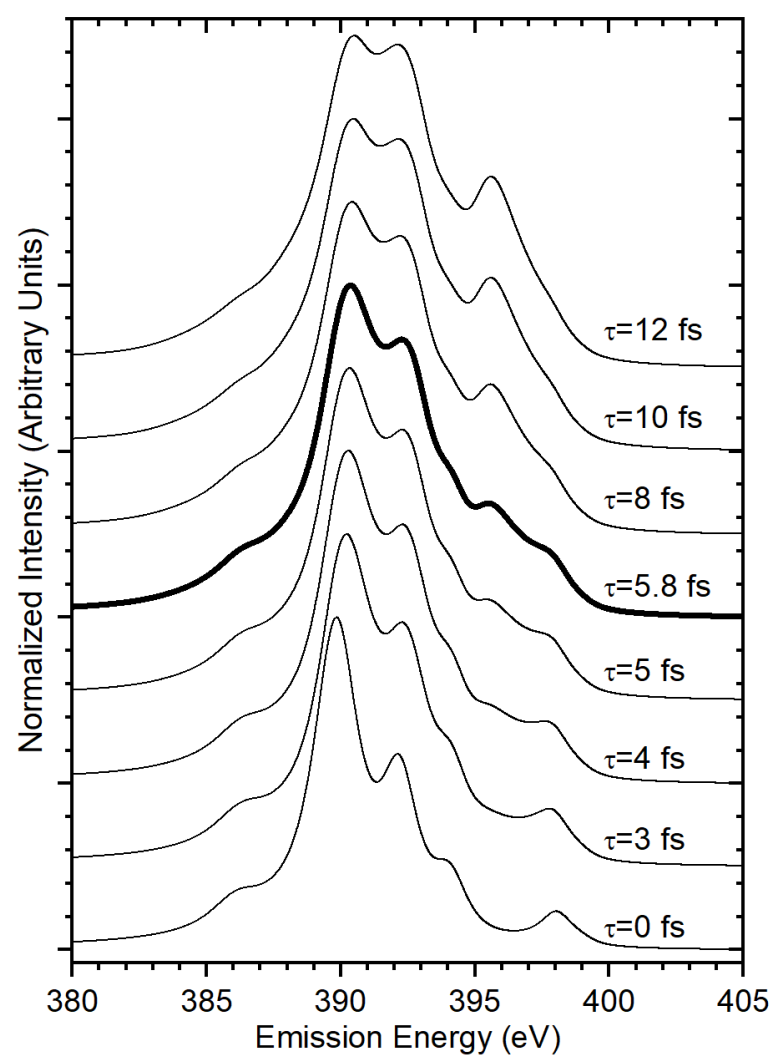

Figure S5. Simulated N K XES spectra, including ultrafast dynamics for different core-level lifetimes $(\tau)$. The main text comparisons are based on the $\tau=5.8 \mathrm{fs}$ spectrum. All spectra were normalized to maximum peak height, and vertical offsets were added for clarity.

\section{Sample Preparation}


The sample was an approximately $60 \mathrm{~nm}$ thick film of $\mathrm{CH}_{3} \mathrm{NH}_{3} \mathrm{PbI}_{3-\mathrm{x}} \mathrm{Cl}_{\mathrm{x}}$, prepared under inert atmosphere at Oxford University ${ }^{2}$ and packaged under inert atmosphere for transport to Berlin and, thence, to the Advanced Light Source. The film was introduced into the UHV system via a dry $\mathrm{N}_{2}-$ filled transport chamber, and therefore was never exposed to ambient conditions prior to measurement.

\section{Experiment}

XES spectra were measured at the rollup position of beamline 8.0.1 of the Advanced Light Source, Lawrence Berkeley National Laboratory, with the high-transmission x-ray spectrometer ${ }^{3}$ of the SALSA endstation ${ }^{4}$. The excitation energy was calibrated using the $\mathrm{N}_{2}$ gas absorption (first maximum at $400.88 \mathrm{eV}^{5}$ ), and the emission spectra were calibrated using elastically scattered photons (i.e., the Rayleigh line). To avoid beam-induced damage, the XES spectra were measured while the sample was moved in a direction perpendicular to the incoming photons at a speed of $400 \mu \mathrm{m} / \mathrm{s}$. Given the size of the photon beam, this limits exposure of a given spot of the sample to approximately $0.075 \mathrm{~s}$.

In order to determine the optimal conditions to minimize beam damage, the speed at which the sample was scanned during the measurement was varied, and spectra measured under different conditions were compared. The excitation spot was approximately $30 \mu \mathrm{m} \times 100 \mu \mathrm{m}(\mathrm{H} \times \mathrm{W})$; the vertical scanning speed can thus be used to determine the exposure time of a given area of the sample. On damaged samples, it was observed that a distinct fluorescence signal appeared when exciting well below the expected N $K$-edge absorption onset. Figure S6a shows the fluorescence signal observed when exciting at $399.9 \mathrm{eV}$ at different scanning speeds. The signal intensity decreases significantly as scanning speed increases, with the rate of decrease diminishing at higher speeds. The integral fluorescence count rate in the 380-397 eV region decreases by nearly an order 
of magnitude in the $400 \mu \mathrm{m} / \mathrm{s}$ spectrum compared to the $5 \mu \mathrm{m} / \mathrm{s}$ spectrum, and over 1.5 orders of magnitude compared to the measurement performed on a stationary sample (illuminated for a total of approximately 10 minutes). In the "stationary" (i.e., without scanning) measurement, the preedge intensity is approximately $80 \%$ that of the nonresonantly-excited spectrum. We also find that this measured intensity goes away when exciting at slightly higher energies (Figure S4), and so represents a true pre-edge resonant feature rather than a background signal. Absorption resonances at similar energies have been previously attributed to species with a singly-protonated $\mathrm{N}^{1}$.

Figure S6b shows the nonresonant N K XES spectrum measured in similar fashion, with sample speeds up to $400 \mu \mathrm{m} / \mathrm{s}$. The "stationary" spectrum is clearly no longer an accurate indication of the true $\mathrm{N} K$ XES spectrum of the perovskite film, but rather of molecular fragments.

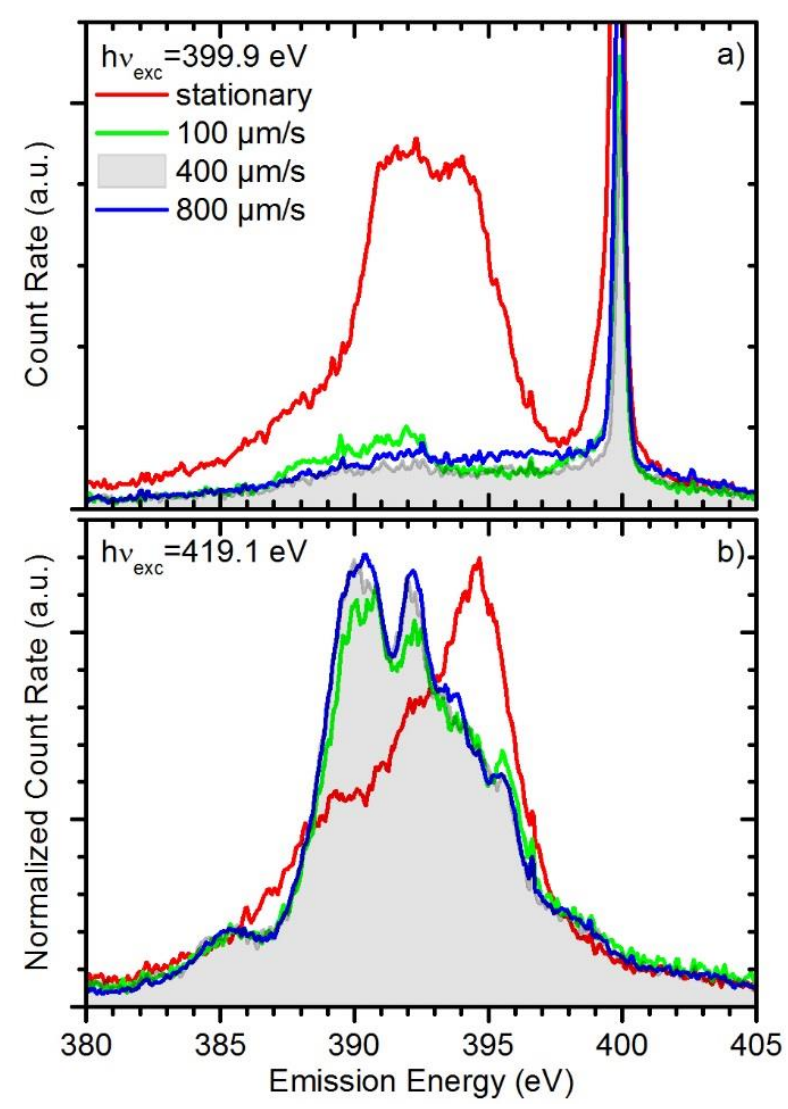

Figure S6. a) resonantly and b) nonresonantly excited $\mathrm{N} \mathrm{K}$ XES spectra of $\mathrm{CH}_{3} \mathrm{NH}_{3} \mathrm{PbI}_{3-\mathrm{x}} \mathrm{Cl}_{\mathrm{x}}$, measured while raster-scanning the sample under the incident beam at different scan rates. Spectra 
labelled "stationary" were measured without scanning, with the beam incident at the same location for $<10 \mathrm{~min}$. For reference, the spectra measured at $400 \mu \mathrm{m} / \mathrm{s}$ are shown with a filled gray area. A background signal containing a small contribution of the Ti $3 \mathrm{~s}-2 \mathrm{p}$ fluorescence transition from the substrate (excited via higher harmonics of the undulator) was subtracted from each spectrum, and the integral intensity of the nonresonant spectra was normalized to unity.

In the spectra recorded while scanning, there is a clear effect of scanning speed upon the shape of the measured spectra, with the two main peaks around $390.5 \mathrm{eV}$ and $392 \mathrm{eV}$ becoming more clearly defined. Above $400 \mu \mathrm{m} / \mathrm{s}$, however, the effect has saturated and the spectra are, within a noise margin, identical. The spectrum shown in main text Figure 1 is the sum of the $400 \mu \mathrm{m} / \mathrm{s}$ spectrum and the two measured at $800 \mu \mathrm{m} / \mathrm{s}$. When exciting on-resonance, as in main text Figure 3, the absorption cross section is approximately doubled, which may lead to slightly increased beam damage, but, based on the scanning-speed dependence, we would not expect a significant effect on the results.

\section{Simulations}

The simulations of XES spectra were based on electronic ground-state calculations in the Quickstep code in the CP2K package ${ }^{6-8}$. To investigate effects of dynamics during the core-hole lifetime, we performed Born-Oppenheimer molecular dynamics (MD) simulations in the CPMD program $^{9}$. Both programs allow for periodic density functional theory (DFT) calculations of large systems, and we employed the gradient-corrected BLYP functional augmented with van der Waals interactions $^{10-12}$.

Nonresonant carbon and nitrogen $K$-edge XES spectra were simulated at configurations before and during the dynamics in the core-ionized state. This classical approach to include effects of the 
finite core-hole lifetime has been valuable for identifying spectral features in studies of XES in solution $^{13-16}$.

In the CPMD program ${ }^{9}$, the Kohn-Sham orbitals and electron density are expressed in a plane wave basis set with kinetic energy cut-offs of 70/280 Ry, in combination with a pseudopotential description of the core-levels. The core levels are only implicitly represented in the plane-wave framework, but core-ionized and core-excited states can be well represented by replacement of the standard pseudopotential on the core-ionized atom with particular pseudopotentials generated with a $1 \mathrm{~s}^{1}$ occupation, as shown in several previous applications ${ }^{13,17,18}$. For the carbon and nitrogen and for the atoms with the $\mathrm{N}$ 1s and $\mathrm{C}$ 1s core holes, we used a Trouiller-Martins norm-conserving pseudopotential expressed in Kleinman-Bylander ${ }^{19-20}$ form. For I and $\mathrm{Pb}$, we used Godecker-TeterHutter (GTH) pseudopotentials ${ }^{21,22}$, and for hydrogen a local pseudopotential with a parameterized Gaussian. The calculations were performed at the $\Gamma$ point.

The starting configurations for the simulation of the XES spectra were taken from the trajectory underlying the sampling in our previous photoelectron spectroscopy investigation of $\mathrm{PbI}_{3} \mathrm{CH}_{3} \mathrm{NH}_{3}(\mathrm{~s})^{23}$. The simulation cell parameters were $\mathrm{a}=\mathrm{b}=12.5176 \AA$ and $\mathrm{c}=12.4444 \AA$. The core-hole lifetimes of nitrogen and carbon $1 \mathrm{~s}$ are $5.8 \mathrm{fs}^{24}$ and $8.4 \mathrm{fs}^{25}$, respectively, hence only short simulations were required; $30 \mathrm{fs}$ with a simulation time-step of $0.1 \mathrm{fs}$.

In the CP2K package ${ }^{6,7}$, we used the implementation of core-level spectroscopy ${ }^{26}$ in Quickstep $^{8}$. In the Gaussian augmented plane-wave approach, the representation of the Kohn-Sham orbitals in a local basis set and the density in a combination of local and plane-wave basis sets allows for allelectron calculations and an explicit representation of the core hole. In electronic ground-state calculations employed for the XES simulations, GTH pseudopotentials ${ }^{21-22}$ and corresponding 
DZVP basis sets were used on all atoms, except for the one atom in the system excited by the xrays, for which the carbon and nitrogen $6-311++\mathrm{G} 2 \mathrm{~d} 2 \mathrm{p}$ basis sets were used.

For comparison to experimental data, the discrete spectra were convoluted with a Gaussian function with a $0.4 \mathrm{eV}$ full-width-at-half-maximum; the results are shown in main text Figure 1. Core-excited states relax by Auger and fluorescence decay at a predictable rate, generally described by the core-hole lifetime, which serves as a time constant in the exponential decay function. The lifetime can be estimated based on its contribution to the spectral broadening, which follows an energy-time formulation of the Heisenberg uncertainty principle; here we use a corehole lifetime of $5.8 \mathrm{fs}$ for the $\mathrm{N} 1 \mathrm{~s}$ core-excited state, ${ }^{24}$ and $8.4 \mathrm{fs}$ for the $\mathrm{C} 1 \mathrm{~s}$ core-excited state ${ }^{25}$. The core-hole lifetime was used to weight each of the calculated spectra in main text Figure $2 b$ to be used in the summation. The relative weight of the $20 \mathrm{fs}$ spectrum is $3.2 \%$ of the $0 \mathrm{fs}$ "groundstate" contribution, demonstrating that the 20 fs time scale is sufficient to describe the effects of proton dynamics on the measurement. To better compare with the measured spectra, a Lorentzian broadening was added. The scale parameter was chosen to have a linear dependence in energy where the slope was fit to match the width of the four peaks in the experimental spectra. This resulted in a FWHM varying between 2.5 and $0.62 \mathrm{eV}$ in the intervals $262-286 \mathrm{eV}$ and $379-403$ $\mathrm{eV}$, respectively. The same dependence, but shifted, was used for both the $\mathrm{N}$ and $\mathrm{C}$ results. The alignment between the calculations and the measurements (Figure 1 and Figure S3) was chosen to maximize agreement. To improve agreement between measurement and model, the bandwidths of the calculated $\mathrm{N}$ and C XES spectra were uniformly stretched by $5.5 \%$ to account for the underestimation of band widths by DFT. 


\section{REFERENCES}

1 R.G. Wilks, J.B. MacNaughton, H.-B. Kraatz, T. Regier, R.I.R. Blyth, and A. Moewes, "A Comparative Theoretical and Experimental Study of the Radiation Induced Decomposition of Glycine” J. Phys. Chem. A 2009, 113, 5360.

2 M.M. Lee, J. Teuscher, T. Miyasaka, T.N. Murakami, and H.J. Snaith, "Efficient Hybrid Solar Cells Based on Meso-Superstructured Organometal Halide Perovskites" Science 2012, 338, 643.

3 O. Fuchs, M. Blum, M. Weigand, E. Umbach, L. Weinhardt, M. Bär, C. Heske, J.D. Denlinger, Y.-D. Chuang, W. McKinney; et al. "High-Resolution, HighTransmission Soft X-ray Spectrometer for the Study of Biological Samples” Rev. Sci. Instrum. 2009, 80, 063103-1-7.

4 M. Blum, L. Weinhardt, O. Fuchs, M. Bär, Y. Zhang, M. Weigand, S. Krause, S. Pookpanratana, T. Hofmann, W. Yang; et al. "Solid And Liquid Spectroscopic Analysis (SALSA) -- a Soft X-ray Spectroscopy Endstation with a Novel FlowThrough Liquid Cell” Rev. Sci. Instrum. 2009, 80, 123102-1-6.

$5 \quad$ R.N.S. Sodhi and C.E. Brion, "Reference Energies for Inner Shell Electron EnergyLoss Spectroscopy” J. Electron Spectrosc. 1984, 34, 363-372.

6 CP2K version 2.6 (Development Version), the CP2K developers group (2000-2014). CP2K is freely available from http://www.cp2k.org/.

7 J. Hutter, M. Iannuzzi, F. Schiffmann, and J. VandeVondele, “CP2K: Atomistic Simulations of Condensed Matter Systems” Wiley Interdiscip. Rev: Comput. Mol. Sci. 2014, 4, 15. 
G. Lippert, J. Hutter, and M. Parrinello, “The Gaussian and Augmented-Plane-Wave Density Functional Method for Ab Initio Molecular Dynamics Simulations” Theor. Chem. Acc. 1999, 103, 124.

9 CPMD, http://www.cpmd.org/, copyright IBM Corp 1990-2008, MPI für Festkörperforschung Stuttgart 1997-2001.

10 A. D. Becke, "Density-Functional Exchange-Energy Approximation with Correct Asymptotic Behavior" Phys. Rev. A 1988, 38, 3098.

11 C. Lee, W. Yang, and R. G. Parr, "Development of the Colle-Salvetti CorrelationEnergy Formula into a Functional of the Electron Density" Phys. Rev. B 1988, 37, 785.

12 S. Grimme, "Semiempirical GGA-type Density Functional Constructed with a LongRange Dispersion Correction” J. Comp. Chem. 2006, 27, 1787.

13 M. Blum, M. Odelius, L. Weinhardt, S. Pookpanratana, M. Bär, Y. Zhang, O. Fuchs, W. Yang, E. Umbach, and C. Heske, "Ultrafast Proton Dynamics in Aqueous Amino Acid Solutions Studied by Resonant Inelastic Soft X-ray Scattering” J. Phys. Chem. B 2012, 116, 13757-13764.

14 M. Odelius, H. Ogasawara, D. Nordlund, O. Fuchs, L. Weinhardt, F. Maier, E. Umbach, C. Heske, Y. Zubavichus, M. Grunze, J.D. Denlinger, L.G.M. Pettersson, and A. Nilsson, "Ultrafast Core-Hole-Induced Dynamics in Water Probed by X-Ray Emission Spectroscopy” Phys. Rev. Lett. 2005, 94, 227401.

15 M. Odelius, "Molecular Dynamics Simulations of Fine Structure in Oxygen-Edge Xray Emission Spectra of Liquid Water and Ice” Phys. Rev. B 2009, 79, 144204. 
16 M. Odelius, "Information Content in O [1s] K-edge X-ray Emission Spectroscopy of Liquid Water" J. Phys. Chem. A 2009, 113, 8176.

17 M. Cavalleri, M. Odelius, A. Nilsson, and L. G. M. Pettersson, "X-ray Absorption Spectra of Water within a Plane-Wave Car-Parrinello Molecular Dynamics Framework” J. Chem. Phys. 2004, 121, 10065.

18 E. M. J. Johansson, M. Hedlund, M. Odelius, H. Siegbahn, and H. Rensmo, "Frontier Electronic Structures of Ru(tcterpy)(NCS $)_{3}$ and $\mathrm{Ru}(\mathrm{dcbpy})_{2}(\mathrm{NCS})_{2}$ : A Photoelectron Spectroscopy Study” J. Chem. Phys. 2007, 126, 244303.

19 N. Troullier and J. L. Martins, "Efficient Pseudopotentials for Plane-Wave Calculations" Phys. Rev. B 1991, 43, 1993.

20 L. Kleinman and D. M. Bylander, "Efficacious Form for Model Pseudopotentials" Phys. Rev. Lett. 1982, 48, 1425.

21 S. Goedecker, M. Teter, and J. Hutter, "Separable Dual-Space Gaussian Pseudopotentials” Phys. Rev. B 1996, 54, 1703.

22 C. Hartwigsen, S. Goedecker, and J. Hutter, "Relativistic Separable Dual-Space Gaussian Pseudopotentials from H to Rn” Phys. Rev. B 1998, 58, 3641.

23 B. Philippe, T.J. Jacobsson, J.-P. Correa-Baena, N.K. Jena, A. Banerjee, S. Chakraborty, U.B. Cappel, R. Ahuja, A. Hagfeldt, M. Odelius; et al. "Valence Level Character in a Mixed Perovskite Material and Determination of the Valence Band Maximum from Photoelectron Spectroscopy: Variation with Photon Energy" J. Phys.

Chem. C 2017, 121 (48), 26655-26666. 
24 B. Kempgens, A. Kivimäki, M. Neeb, H.M. Köppe, A.M. Bradshaw, and J. Feldhaus, "A High-Resolution N 1s Photoionization Study of the $\mathrm{N}_{2}$ Molecule in the NearThreshold Region” J. Phys. B: At. Mol. Opt. Phys. 1996, 29, 2389-5402.

25 H.M. Köppe, A.L.D. Kilcoyne, J. Feldhaus, and A.M. Bradshaw, "Relaxation Effects in C 1s Photoionisation of CO: A High Resolution Photoelectron Study in the NearThreshold Region” J. Electron Spectrosc. 1995, 75, 97-108.

26 M. Iannuzzi and J. Hutter, "Inner-Shell Spectroscopy by the Gaussian and Augmented Plane Wave Method" Phys. Chem. Chem. Phys. 2007, 9, 1599. 